\title{
Percepción social sobre la gestión de residuos urbanos: el caso del municipio de Puçol (Valencia)
}

\author{
Víctor Agulló CALATAYUd ${ }^{1}$ \\ victor.agullo@uv.es \\ Gregorio GonZÁLEZ ALCAIDE ${ }^{2}$ \\ gregorio.gonzalez@uv.es \\ Carlos ABELlÁN ANDRÉS 3 \\ carlosabellan@uv.es
}

Departament de Sociologia i Antropologia Social-Universitat de València

Facultat de Ciències Socials

Recibido: 20 de Marzo de 2011

Enviado a evaluar: 21 de Marzo de 2001

Aceptado: 5 de Octubre de 2011

\section{RESUMEN}

El presente estudio analiza la situación de la gestión de los residuos y la problemática medioambiental del municipio de Puçol (Valencia) a partir del análisis estadístico multivariante de datos de una encuesta sociológica. Se ponen de manifiesto los siguientes aspectos: a) El porcentaje de población que recicla correctamente todo tipo de residuos es únicamente del $8,93 \%$ y el porcentaje de personas que reciclan algún tipo de residuo es del 60,97\%; b) tener un alto nivel de estudios y el hecho de disponer de contenedores cercanos conlleva que, de forma significativa, se recicle más y mejor; c) el contenedor de plásticos, bricks y latas no es utilizado apropiadamente, dado que más del $70 \%$ de la muestra utiliza el contenedor de basura orgánica para este fin; y d) la población de Puçol percibe la falta de conciencia ambiental como el principal problema medioambiental del municipio, con un porcentaje del $36,3 \%$.

Palabras clave: Gestión de los residuos, medio ambiente, percepción social, encuesta ciudadana.

\section{Social perception of environment and waste management in Puçol (Valencia-Spain)}

\begin{abstract}
The present study analyzes the situation of waste management and environmental problems in the municipality of Puçol (Valencia-Spain) from multivariant statistical analysis data from a sociological survey. It can be highlighted the following aspects: to) The citizen percentage that recycles correctly all type of waste resi-

1 Departament de Sociologia i Antropologia Social. Universitat de València.

2 Departament d'Història de la Ciència i Documentació. Universitat de València.

3 Àrea de Genòmica i Salut. Centre Superior d'Investigació en Salut Pública (CSISP). Generalitat Valenciana


dues is of $8.93 \%$ and the percentage of recyclers of any kind of residue is of $60.97 \%$; b) to have a high educational level and the fact of having close containers entails, in a significant way, to recycled more; c) the container of plastics, bricks and cans is not used appropriately given that more than $70 \%$ of the sample uses the organic garbage container for this purpose; and d) the town of Puçol perceives the lack of environmental awareness as the environmental main problem of the municipality with a percentage of $36.3 \%$.

Keywords: Waste management, social perception, environment, sociological study, citizen survey, home waste.

\section{La présente recherche analyse la situation de la gestion des résidus et la pro- blématique environnementale de la ville de Puçol (Valence-Espagne)}

\section{RESUMÉ}

A partir de l'analyse statistique multivariante d'une enquête sociologique. Ils peuvent êtres mis en évidence les suivants aspects: à) Le pourcentage de population que recycle correctement tout genre de résidus est uniquement de $8,93 \%$ et le pourcentage de personnes qui recyclent quelque type de résidu est de $60,97 \%$; b) avoir un haut niveau d'études et le fait de disposer de bennes proches à la maison comporte que, de façon significative, il se recycle plus et mieux; c) la benne de plastiques, bricks et boîtes de conserve n'est pas utilisé adéquatement, étant donné que plus de $70 \%$ de l'échantillon utilise la benne à ordures organique pour cette finalité; et d) la population de Puçol perçoit la faute de conscience environnementale comme le principal problème environnemental de la commune, avec un pourcentage de $36,3 \%$.

Mots clé: Gestion des résidus, environnement, perception sociale, enquête citoyenne.

\section{INTRODUCCIÓN}

Las sociedades modernas han vivido históricamente confiadas en la idea de un progreso continuo y permanente, donde los recursos eran abundantes e ilimitados. Los núcleos urbanos, desde su fundación en época romana, se han dotado de mecanismos para dar respuesta al tratamiento de los desechos urbanos (Dupré Raventós y Remolà Vallverdú, 2002). Sin embargo, el gran crecimiento demográfico y la progresiva urbanización de las modernas sociedades globales, conlleva un notable incremento en la generación de residuos (Hernàndez Martí, 2002), tendencia que se ve acrecentada con la instauración de la cultura del "usar y tirar", con la rápida obsolescencia de muchos artículos de uso doméstico y con el volumen creciente de envases y embalajes de todo tipo (García, 2006), que hacen cada vez más necesario considerar los cambios en los procesos productivos, en el tipo de productos, en las formas de comercialización y en el consumo, así como en el sentir y actuar de la sociedad, pues ha ido surgiendo una racionalidad ambiental que reclama sea respetado el medio ambiente (Velázquez Patiño, 2008). En ese sentido, los residuos urbanos se erigen como uno de los ámbi- 
tos en que la predisposición favorable a hacer algo para proteger el medio ambiente está más ampliamente difundida entre la población (García, 2006).

Lograr una gestión sostenible de los residuos domésticos se ha convertido en uno de los grandes desafíos y prioridades con los que se enfrenta cualquier acción de gobierno, muy especialmente en el caso de los ayuntamientos, puesto que se trata de una problemática ciudadana que afecta muy especialmente al ámbito local. Diversos estudios han señalado que la tutela ambiental municipal, a través de la atribución legal de competencias en materia de recogida y tratamiento de basuras (Tortosa, Miralles y Lostado, 1996), es la vía más adecuada para solventar los problemas derivados de la degradación del ecosistema urbano (Jiménez, 2006), como así lo pone de manifiesto el estudio Ecología y Medio Ambiente del Centro de Investigaciones Sociológicas (2007), según el cual, el 30,7\% de la ciudadanía española piensa que es el ayuntamiento la institución a la que le corresponde la mayor responsabilidad a la hora de hacer frente a los problemas medioambientales existentes en su pueblo o ciudad, por delante del Ministerio de Medio Ambiente (17,9\%); la Comunidad Autónoma (9,3\%); los propios ciudadanos $(8,5 \%)$ o las empresas e industrias $(4,1 \%)$.

En el País Valenciano, se constata un progresivo aumento del reciclaje que ha pasado de 116.863 toneladas en 2006 a 130.273 toneladas de envases ligeros (envases de plástico, latas y briks) y envases de cartón y papel en 2010, de acuerdo con los datos del Sistema Integrado de Gestión (SIG) de Ecoembes. Así, cada valenciano depositó de media en el contenedor amarillo 8 kilogramos de residuos al año y 6 kilos de envases de cartón al contenedor azul.

Los estudios sociológicos vinculados con la recogida selectiva de los residuos tienen por objeto detectar el grado de colaboración ciudadana y la incidencia de las distintas campañas de concienciación. El objetivo del presente trabajo consiste en analizar las características sociodemográficas que inciden en la gestión de los residuos urbanos y la problemática medioambiental a partir un análisis estadístico multivariante de datos de encuesta tomando como referente el caso del municipio valenciano de Puçol.

\section{METODOLOGÍA}

Puçol es un municipio costero de 17.249 habitantes situado en la comarca de L'Horta Nord, en el Noroeste de la provincia de Valencia, de cuya capital dista 20 kilómetros. Para analizar las problemáticas medioambientales y la gestión de los residuos en el municipio se diseñó una encuesta dirigida al conjunto de la población mayor de 15 años con datos del patrón de habitantes a fecha de 1 de Enero de 2010. Con el propósito de obtener una información representativa se llevó a cabo un muestreo no probabilístico estratificado con la selección de las unidades primarias de muestreo (sexo) y de las unidades secundarias (grupos quinquenales de edad, o nacionalidad en el caso de los inmigrantes) de forma aleatoria proporcional a partir de un nivel de confianza del 95,5\% (dos sigmas), y $\mathrm{P}=\mathrm{Q}$, siendo el error de $\pm 5 \%$ para el conjunto de la muestra autóctona y de $\pm 10 \%$ para la población inmigrante del municipio de las nacionalidades más representativas.

La muestra final quedó compuesta por 453 personas: 374 personas autóctonas y 79 inmigrantes. Las principales nacionalidades del municipio de la muestra queda- 
ron representadas por 46 personas ( 26 hombres y 20 mujeres) con la siguiente distribución muestral: Marruecos (45,7\%), Rumanía (21,7\%), Ecuador (17,4\%), Colombia (6,5\%) y China y Bolivia (ambas con el 4,3\%, respectivamente).

Se diseñó una batería de preguntas que recogían información sobre la percepción ciudadana de los principales problemas medioambientales del municipio; así como para obtener información acerca de diversos aspectos relacionados con las creencias y actitudes acerca de la gestión de los residuos, entre ellos, el porcentaje y las características sociodemográficas de la población que recicla adecuadamente, las principales causas que dificultan la recogida de los residuos o una valoración sobre los recursos municipales para la recogida de residuos urbanos, entre ellos la tasa impositiva por la recogida de basura municipal, el conocimiento y la utilización del Ecoparque - creado para la gestión de los residuos sólidos urbanos del municipio-, y la frecuencia de recogida de los mismos, el número, la ubicación y el mantenimiento de los contenedores.

Previamente a la realización de la encuesta definitiva se midió tanto la fiabilidad del cuestionario como su consistencia interna, a partir del cálculo del alfa de Cronbach con los ítems de cada subescala o dimensión. Se pasó un pretest a 20 personas del municipio y dos preguntas que no se comportaban adecuadamente fueron reformuladas en el cuestionario para garantizar en todo momento que todas las preguntas tuvieran sentido, provocaran las respuestas esperadas y la categorización fuera la correcta.

\section{RESULTADOS}

El porcentaje de población que recicla correctamente todo tipo de residuos fue únicamente del $8,93 \%$, si bien, el porcentaje de personas que reciclan algún tipo de residuo se situó en el $60,97 \%$. Se determinó que el nivel de estudios y el hecho de tener contenedores cerca de casa son las dos variables que influyen de forma significativa en el hecho de que se recicle y que se haga correctamente.

El 36,3\% de la población de Puçol percibe la falta de conciencia ambiental como el principal problema medioambiental del municipio. Asimismo, más de un tercio de la muestra $(34,5 \%)$ señala la desaparición de la huerta como el segundo problema del mismo. También son destacados como problemáticos la limpieza de las calles y la calidad del agua de consumo, entre otros aspectos (tabla 1).

Tabla 1. Principales problemas medioambientales de Puçol.

\begin{tabular}{|l|c|}
\hline \multicolumn{1}{|c|}{ Problemas medioambientales } & $\%$ \\
\hline 1 La falta de conciencia medioambiental & 36,3 \\
\hline 2 La desaparición de l'horta & 34,5 \\
\hline 3 La limpieza de las calles & 31,1 \\
\hline 4 La calidad del agua de consumo & 28,9 \\
\hline 5 La gestión y recogida de los residuos & 21,6 \\
\hline 6 El crecimiento urbanístico & 17,4 \\
\hline 7 Los incendios o la destrucción de los espacios naturales & 17,1 \\
\hline 8 La falta de jardines y zonas verdes & 16,6 \\
\hline 9 El ruido excesivo & 16,3 \\
\hline 10 La contaminación atmosférica & 13,9 \\
\hline
\end{tabular}


En relación con la gestión de los residuos, la práctica totalidad de los vecinos del municipio (99,5\%) manifiesta que cuenta con contenedores de basura orgánica a menos de cien metros de sus casas. Por el contrario, más de la mitad de los encuestados $(55,6 \%)$ señala que no disponen de contenedores de reciclado de plástico a menos de cien metros de sus hogares y aproximadamente la mitad de los vecinos no dispone de contenedores de vidrio $(51,1 \%)$ y de papel $(49,7 \%)$ próximos a sus hogares (tabla 2$)$.

Tabla 2. Contenedor cerca de casa (menos de $100 \mathrm{~m}$ ) para la recogida de los residuos.

\begin{tabular}{|c|c|c|}
\hline Tipo de contenedor & Sí & No \\
\hline Orgánica & $99,5 \%$ & $0,5 \%$ \\
\hline Plástico & $42,6 \%$ & $57,4 \%$ \\
\hline Papel & $50,3 \%$ & $49,7 \%$ \\
\hline Vidrio & $48,9 \%$ & $51,1 \%$ \\
\hline
\end{tabular}

El contenedor de plásticos presenta el peor uso, ya que más del 70\% utilizan el contenedor de basura orgánica para depositar estos elementos. En cuanto al reciclaje del papel y cartón, un $39,5 \%$ utiliza el contenedor de basura orgánica. Un comportamiento similar se observa en relación con el vidrio, ya que el 39,2\% de las personas encuestadas manifiesta que deposita estos elementos en el contenedor de residuos orgánicos (tabla 3).

Tabla 3. Uso correcto del contenedor por tipo de residuos.
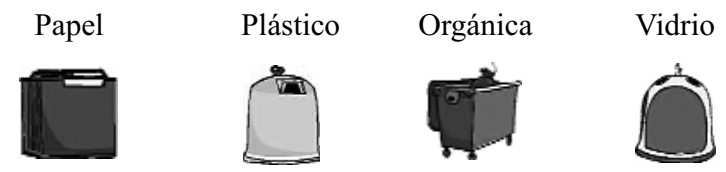

\begin{tabular}{|l|c|c|c|c|}
\hline Cartón y papel (periódicos, revistas) & $59,2 \%$ & $1,1 \%$ & $39,5 \%$ & $0,6 \%$ \\
\hline Botellas y botes de vidrio & $1,7 \%$ & $1,1 \%$ & $39,2 \%$ & $58 \%$ \\
\hline Restos de comida & $1,7 \%$ & $0,8 \%$ & $97,8 \%$ & $0,8 \%$ \\
\hline Botes refresco, comida, bolsas... & $1,4 \%$ & $21 \%$ & $77,8 \%$ & $1,7 \%$ \\
\hline Bricks de leche, zumo & $3,6 \%$ & $22,9 \%$ & $73,5 \%$ & $0,9 \%$ \\
\hline
\end{tabular}

La principal causa que dificulta el reciclaje de los residuos $(35,1 \%)$ es el hecho de no disponer de los contenedores adecuados cerca de casa. Asimismo, para el $29,5 \%$ de los encuestados, separar los residuos en casa resulta incómodo. Los que opinan que no sirve para nada suponen el $13,1 \%$ mientras que los que dicen no disponer de tiempo representan el $8,6 \%$ de la muestra (figura 1 ). 
Figura 1. Causas que dificultan el reciclaje de los residuos

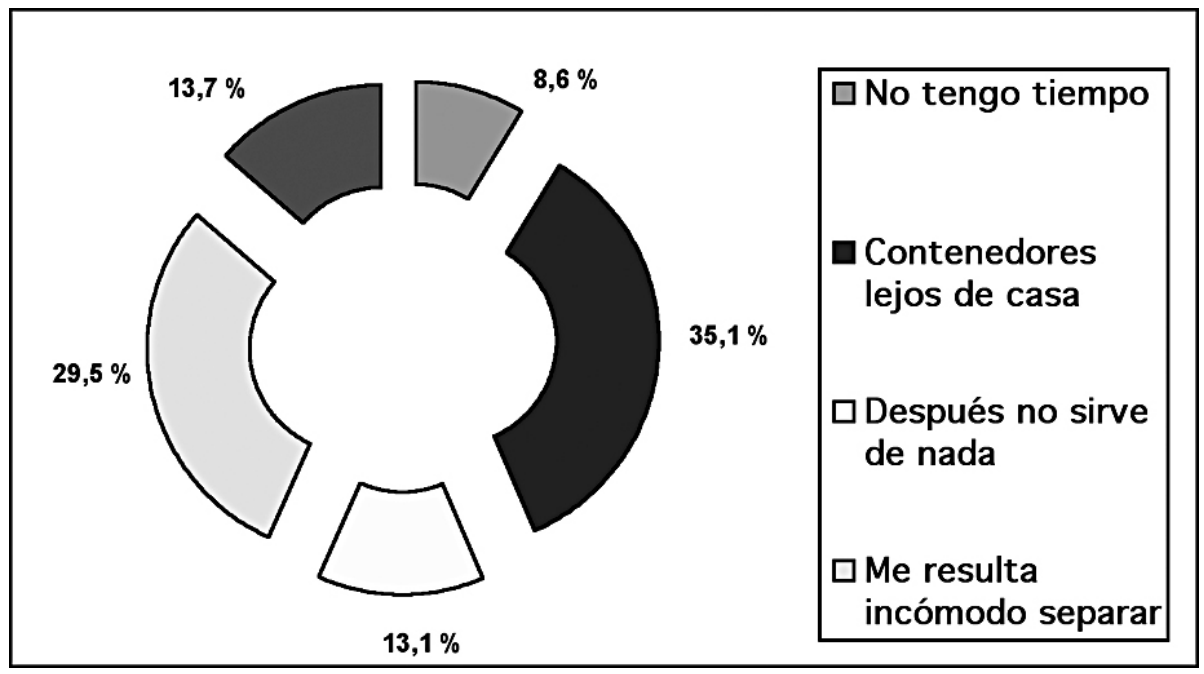

Para el $81,6 \%$ de la muestra, la frecuencia de recogida de residuos urbanos es buena o muy buena, y solamente para el $12,6 \%$ es regular y para el 5,8\% mala. Sin embargo, los ítems número de contenedores, mantenimiento y ubicación de los mismos no obtienen una valoración tan positiva. Para el 45,53\% de los encuestados, el número de contenedores no es el adecuado y su mantenimiento es valorado como regular o malo por el 53,37\%.

El $77,2 \%$ de la muestra conoce tanto la existencia como el funcionamiento del Ecoparque y un 56,8\% lo utiliza. Los principales productos que se depositan en este centro son, por este orden, electrodomésticos, muebles y escombros. El $70 \%$ de la muestra llama al Ayuntamiento si quiere deshacerse de algún mueble y el 26,1\% lo lleva al Ecoparque (figura 2).

Figura 2. Modo para deshacerse de los muebles

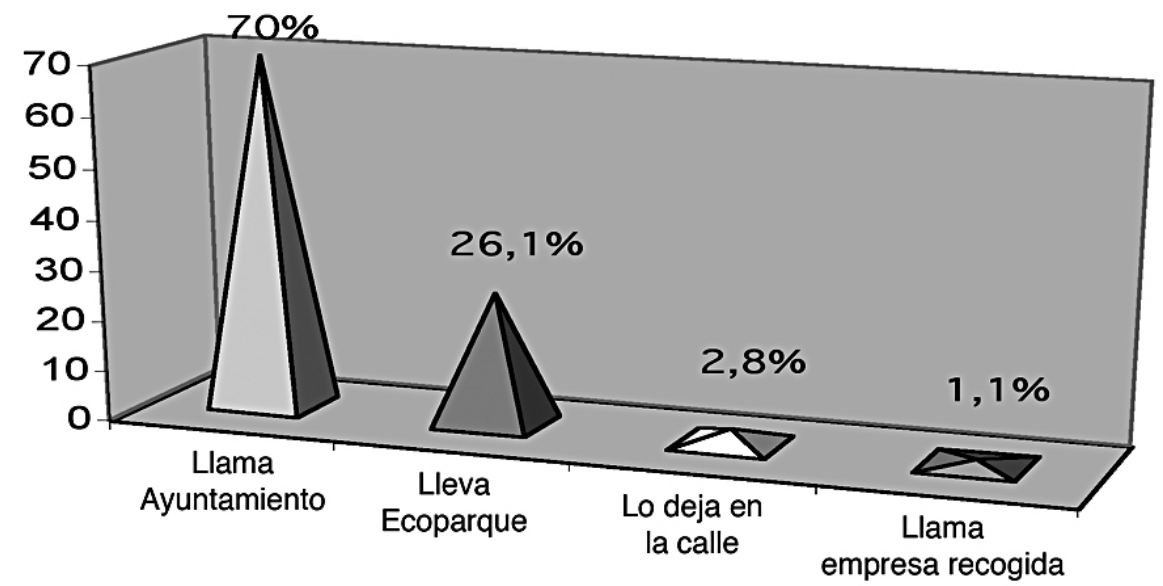


El 50,6\% de la muestra está de acuerdo con la tasa impositiva municipal de recogida de basuras, mientras que el $47,9 \%$ está en desacuerdo o muy en desacuerdo. Cuando se invita a los ciudadanos de Puçol a que expresen abiertamente sus opiniones acerca de la gestión de los residuos en la localidad, se vuelve a señalar la falta de contenedores de todos los tipos como uno de los principales problemas. También hay quejas referidas a la de limpieza de los contenedores en verano, a las molestias que ocasionan a los peatones y niños los excrementos caninos y la falta de conciencia cívica por parte de sus dueños. Otros aspectos señalados son la necesidad de aumentar las calles peatonales, la fumigación contra cucarachas y ratas, que el Ecoparque abra los domingos, que se limpien con mayor frecuencia los barrancos o la implementación de campañas o actividades dirigidas tanto a población adulta como escolar para favorecer una mayor concienciación para que la gente recicle. A diferencia de la población autóctona, la población inmigrante de Puçol considera el ruido excesivo y la calidad del agua de consumo como los problemas medioambientales más importantes. No obstante, la mitad de los ítems presentan porcentajes similares en ambas poblaciones (tabla 4).

Tabla 4. Comparativa principales problemas mediambientales de Puçol.

\begin{tabular}{|l|c|c|}
\hline \multicolumn{1}{|c|}{ Problemas medioambientales } & $\%$ Puçol & \% Inmigrantes \\
\hline 1 La falta de conciencia medioambiental & 36,3 & 34,8 \\
\hline 2 La desaparición de l'horta & 34,5 & 19,6 \\
\hline 3 La limpieza de las calles & 31,1 & 30,4 \\
\hline 4 La calidad del agua de consumo & 28,9 & 41,3 \\
\hline 5 La gestión y recogida de los residuos & 21,6 & 21,7 \\
\hline 6 El crecimiento urbanístico & 17,4 & 15,2 \\
\hline 7 Los incendios o la destrucción de los espacios naturales & 17,1 & 6,5 \\
\hline 8 La falta de jardines y zonas verdes & 16,6 & 28,3 \\
\hline 9 El ruido excesivo & 16,3 & 41,3 \\
\hline 10 La contaminación atmosférica & 13,9 & 2,2 \\
\hline
\end{tabular}

El porcentaje de población inmigrante que afirma reciclar algún tipo de residuo $(60,4 \%)$ es similar al observado en la población autóctona de Puçol. Sin embargo, se ha estimado que el porcentaje de población inmigrante que recicla correctamente todo tipo de residuos es de un $26,67 \%$, lo que supone un $17,74 \%$ más que la población autóctona. Estos porcentajes se distribuyen de forma desigual por países, con un porcentaje mayor de inmigrantes de Rumanía, Bolivia, Colombia y China que declaran que reciclan frente a los que no lo hacen, a diferencia de lo que ocurren en los casos de Marruecos y Ecuador, que es el país que presenta una diferencia más acusada en relación con el hábito de reciclar (figura 3). 


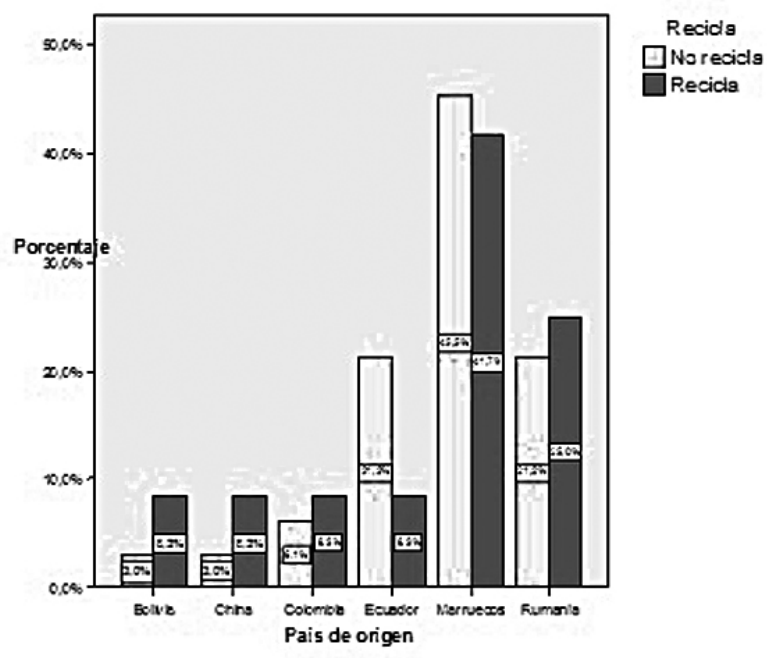

Finalmente, se implementa una regresión logística con la intención de estudiar la influencia sobre la probabilidad de reciclar algún tipo de residuo surgiendo un modelo con los siguientes factores sociodemográficos: nivel de estudios, lengua vehicular y causas que dificultan el reciclaje. Se obtiene que, respecto al nivel de estudios, si la población tiene estudios primarios la probabilidad de reciclar aumenta de forma significativa (pvalor $=0.000194$ ), lo mismo ocurre si se posee estudios secundarios (pvalor $=0.000171)$ y estudios universitarios (pvalor $=0.0000545)$. Igualmente, parece observarse una ligera tendencia por la que las personas valencianohablantes tenderían a reciclar más. Por último, respecto a las causas que dificultan el reciclaje, de nuevo se pone de manifiesto que el hecho de tener los contenedores lejos de casa influye negativamente y de manera significativa en la probabilidad de reciclar algún tipo de residuo.

\section{DISCUSIÓN}

La adecuada gestión de los residuos, junto con el control del consumo de los recursos naturales, la aplicación de políticas de eficiencia energética y la reducción de las emisiones atmosféricas, constituyen los pilares esenciales que deben asegurar lo que se ha definido con el concepto de "sostenibilidad", entendido como el equilibrio dinámico entre las actividades humanas y el medio en el que éste vive y desarrolla sus actividades, asegurando la reducción al mínimo del impacto ambiental (Alejandre Martín, 2000; Otero Pastor, 1998).

Factores como los procesos de concentración urbana y la modificación de los patrones productivos, de comercialización y consumo, han incrementado exponencialmente la generación de residuos que no pueden ser degradados de forma natural en un corto período de tiempo y que además, en ocasiones, pueden suponer graves problemas, como la contaminación del medio ambiente. Frente a esta situación, el 
reciclaje puede constituirse en uno de los mecanismos más eficaces para asegurar la sostenibilidad en relación con la gestión de los residuos, pese a que su importancia sea todavía moderada en Europa en relación con la práctica de enterrar los residuos (Stanners y Bordeau, 1995).

En este sentido, la participación ciudadana es fundamental para asegurar el éxito de los programas de reciclaje, de ahí el interés de los estudios que tratan de determinar cuáles son las actitudes, los comportamientos y la valoración de los ciudadanos en relación con el medio ambiente, y sobre todo, la identificación de las variables que inciden de forma directa en el reciclaje, ya que deben ser tomadas como referentes para la puesta en marcha de las políticas de reciclaje adecuadas o de cara a implementar las medidas correctoras necesarias (Esteban Curiel, 2000).

Numerosos estudios han constatado la existencia de una asociación positiva entre el reciclado y variables sociodemográficas como la edad, el género, el nivel de estudios o el nivel de renta, si bien, de forma más puntual, en otros trabajos no se ha establecido esta relación (para una excelente síntesis al respecto, véase Díaz Meneses y Beerli Palacio, 2006). En el estudio realizado en Puçol, se ha identificado el nivel de estudios como variable sociodemográfica que influye significativamente en el comportamiento del reciclado, ya que a mayor nivel de estudios, mayor probabilidad de reciclar algún tipo o todo tipo de residuos y de realizarlo correctamente. Diversos trabajos sitúan esta variable entre las que más favorecen el reciclaje (Aragonés y Amérigo Cuervo-Arango, 1991; Esteban Curiel, 2000). La asociación positiva entre el nivel de estudios y el reciclaje, se fundamenta en el hecho de que estas personas están más informadas y que poseen una mayor conciencia ecológica y acerca de los problemas medioambientales y de los beneficios que puede aportar el reciclado (Fraj Andrés, 2003; Garcés, Pedraja y Rivera, 1995; Díaz Meneses y Beerli Palacio, 2006). Se puede concluir, por tanto, que fortalecer la conciencia ecológica en la educación formal e incidir en campañas de concienciación o planes de educación ambiental entre las personas de mayor edad que no han podido acceder a la misma y entre las personas de menor nivel educativo, resultan fundamentales para favorecer la conciencia ambiental y el compromiso y la costumbre de reciclar (Díaz Meneses, Beerli Palacio y Martín Santana, 2004), campañas de educación en las que se debería incidir en un uso adecuado de las infraestructuras de recogida selectiva de residuos, ya que éste constituye un aspecto problemático según se ha observado en el estudio realizado en relación con el papel, el plástico y el vidrio.

Otros trabajos también han correlacionado positivamente el hábito del reciclado con determinadas características no analizadas en el presente trabajo, como la personalidad, los valores y estilos de vida y las actitudes de los individuos, entre otras (Corraliza Rodríguez y Martín, 2000; Fraj Andrés, Martínez Salinas y Grande Esteban, 2004).

Por otra parte, el estudio realizado ha puesto de manifiesto que para aumentar el reciclaje y ampliar la recogida selectiva de residuos, resulta fundamental extender la infraestructura de recogida, insuficiente para la mitad de la población estudiada, algo que parece lógico, ya que difícilmente se puede exigir a los ciudadanos un comportamiento si no se facilitan las condiciones materiales para ello, especialmente tenien- 
do presente que un elevado porcentaje de encuestados manifiesta que no recicla porque tiene los contenedores lejos de casa y que percibe la gestión de los residuos como algo incómodo. En este caso como factores interpretativos y explicativos se ha planteado que el esfuerzo que supone o las limitaciones de espacio condicionan la actitud del reciclaje (Franco y Huerta, 1996). En relación con este aspecto, hay una coincidencia en los estudios que han analizado las características situacionales (proximidad de los puntos de recogida, frecuencia y buen estado de los mismos) en la valoración de que resultan fundamentales (Díaz Meneses y Beerli Palacio, 2006), pudiéndose citar a título de ejemplo en relación con los contenedores, el caso exitoso de municipios como Hannover, en el que se adoptó la medida de entregar un contenedor de residuos para cada domicilio con un tamaño adaptado a las necesidades de cada familia (Velázquez Patiño, 2008). En este caso y en el de otras muchas ciudades de países europeos, se han adoptado también otras medidas, como el cobro proporcional por la gestión de los residuos, según la producción de cada casa y en función de las condiciones en la que los residuos son entregados al sistema de recolección, factores que han contribuido en gran medida al incremento en la toma de conciencia por parte de los ciudadanos, no únicamente en relación con la separación de los residuos en el momento de desprenderse de ellos, sino como consumidores en relación con los productos adquiridos (Velázquez Patiño, 2008).

El análisis de la percepción social de la gestión de los residuos no puede desligarse de las distintas problemáticas medioambientales observadas en la población de Puçol que tienen su correspondencia con la creciente sensibilización ambiental ciudadana que ya se viene manifestando desde tiempo atrás a escala europea o estatal, si bien por detrás de los problemas económicos y sociales que inciden más directamente en la vida cotidiana de los individuos, especialmente en coyunturas de crisis económica como la actual (Esteban Curiel, 2000). Finalmente, hay que apuntar que el hecho de que en el presente estudio se hayan detectado diferencias en relación con el hábito de reciclar entre los inmigrantes de distintos países, unido al peso relativo cada vez mayor que la población extranjera ha ido adquiriendo en todos los municipios españoles, nos alerta acerca de la necesidad de considerar esta variable en los estudios, ya que probablemente sea necesario implementar políticas específicas en función de los diferentes colectivos poblacionales, teniendo en cuenta el hecho de que habitualmente los inmigrantes presentan particularidades en cuanto a su estructura demográfica, costumbres y zonas de residencia, variables que además pueden diferir en función de las áreas geográficas o países de procedencia (Alcolea Moratilla, 2000).

Las principales conclusiones del estudio realizado son las siguientes: a) El porcentaje de población que recicla correctamente todo tipo de residuos es únicamente del $8,93 \%$, con un $60,97 \%$ de personas que reciclan algún tipo de residuo; b) tener un alto nivel de estudios y el hecho de disponer de contenedores cercanos son las dos variables que, de forma significativa, conllevan que se recicle más y mejor, por lo que resulta fundamental para favorecer el reciclaje incidir en las políticas educativas y las campañas de concienciación ambiental y extender la mejorar la infraestructura de recogida urbana de residuos; c) el contenedor de plásticos, bricks y latas no es utilizado apropiadamente, dado que más del $70 \%$ de la muestra utiliza el contenedor de basura orgá- 
nica para este fin; también los contenedores de vidrio y papel presentan una utilización deficiente, ya que aproximadamente en un $40 \%$ de los casos estos elementos se depositan en el contenedor de residuos orgánicos; y d) la población de Puçol percibe la falta de conciencia ambiental como el principal problema medioambiental del municipio, con un porcentaje del $36,3 \%$. Asimismo, aproximadamente un tercio de la muestra señala la desaparición de la huerta como el segundo problema en importancia. Sin embargo, para la población inmigrante del municipio, el ruido excesivo y la calidad del agua de consumo como los problemas medioambientales más importantes.

\section{BIBLIOGRAFÍA}

ALCOLEA MORATILLA, MIGUEL ÁNGEL (2000): Análisis espacial y medioambiental de la inmigración en el municipio de Madrid, en: Observatorio Medioambiental, $n^{\circ} 3$, pp. 53-75, Universidad Complutense de Madrid: Instituto Universitario de Ciencias Ambientales, Madrid.

ALEJANDRE MARTÍN, CARLOS (2000): Urbanismo, energía y medio ambiente, en: Observatorio Medioambiental, $\mathrm{n}^{\circ}$ 3, pp. 401-422, Universidad Complutense de Madrid: Instituto Universitario de Ciencias Ambientales, Madrid.

ARAGONÉS, J. IGNACIO; Y AMÉRIGO CUERVO-ARANGO, MARÍA (1991): Un estudio empírico sobre las actitudes ambientales, en: Revista de Psicología Social, vol. 6, no 2, pp. 223-240, Fundación Infancia y Aprendizaje, Madrid.

CENTRO DE INVESTIGACIONES SOCIOLÓGICAS (CIS) (2007); Estudio 2682: Ecología y Medio Ambiente (III). CIS, Madrid, 2007.

CORRALIZA RODRÍGUEZ, JOSÉ ANTONIO; Y MARTÍN, ROCÍO. (2000): Estilos de vida, actitudes y comportamientos ambientales, en: Medio Ambiente y Comportamiento Humano, vol. 1, $\mathrm{n}^{\mathrm{o}}$ 1, pp. 31-56, Universidad de La Laguna: Facultad de Psicología, Santa Cruz de Tenerife.

DÍAZ MENESES, G.; BEERLI PALACIO, A.; Y MARTÍN SANTANA, J. D. (2004): El modelo de hábito de reciclado según el perfil sociodemográfico de los consumidores, en: XVI Encuentro de Profesores Universitarios de Marketing, pp. 191-207. ESIC Editorial, Madrid

DÍAZ MENESES, G.; Y BEERLI PALACIO, A. (2006): El proceso de adopción de la conducta de reciclado: modelos explicativos y variables moderadoras, en: Cuadernos de Economía y Dirección de la Empresa, n 28 , pp. 55-86, Asociación Científica de Economía y Dirección de la Empresa, Madrid.

DUPRÉ RAVENTÓS, XAVIER; Y REMOLÀ VALLVERDÚ, JOSEP ANTÓN (2002): A propósito de la gestión de los residuos urbanos en "Hispania", en: Rómula, $\mathrm{n}^{\circ}$ 1, pp. 39-56, Universidad Pablo de Olavide, Sevilla

ECOEMBES (2010): Ecoembalajes España S.A. Informe Anual 2010.

ESTEBAN CURIEL, GEMA. (2000): Actitudes de los españoles ante los problemas ambientales, en: Observatorio Medioambiental, $\mathrm{n}^{\circ}$ 3, pp. 107-122, Universidad Complutense de Madrid: Instituto Universitario de Ciencias Ambientales, Madrid.

FRAJ ANDRÉS, ELENA (2003): Las variantes demográficas y socioeconómicas como determinantes del comportamiento de reciclaje: su importancia sobre la 
gestión de residuos sólidos, en: Revista de Gestión Pública y Privada, $\mathrm{n}^{\circ}$ 8, pp. 103-118, Escuela Universitaria de Estudios Empresariales de Huesca, Huesca.

FRAJ ANDRÉS, ELENA; MARTÍNEZ SALINAS, EVA; Y GRANDE ESTEBAN, ILDEFONSO. (2004): Un estudio exploratorio sobre las variables psicográficas que influyen en el comportamiento del consumidor ecológico, en: Revista de Economía y Empresa, vol. 21, n 50, pp. 61-88, Academia Europea de Dirección y Economía de la Empresa, Madrid.

FRANCO, JUAN F.; Y HUERTA, EMILIO. (1996): Determinantes de la participación ciudadana en programas de reciclaje de residuos sólidos urbanos, en: Investigaciones Económicas, vol. 20, $\mathrm{n}^{\mathrm{o}}$ 2, pp. 271-280, Fundación Empresa Pública, Madrid.

GARCÉS, C.; PEDRAJA, M. Y RIVERA, P. (1995): Variables sociodemográficas determinantes del comportamiento ecológico de los españoles, en: VII Encuentro de profesores universitarios de marketing, Barcelona, pp. 513-521, ESIC Editorial, Madrid.

GARCÍA, ERNEST (2006): Consumo y medio ambiente en el País Valenciano 19802000, en: Papers: Revista de Sociología, $\mathrm{n}^{\circ}$ 82, pp. 97-120, Universitat Autònoma de Barcelona, Barcelona.

HERNÀNDEZ MARTÍ, GIL-MANUEL (2002); La modernitat globalitzada: anàlisi de l'entorn social, Tirant lo Blanch, Valencia, 2002

JIMÉNEZ, CARMEN (2006); La gestión de residuos en los municipios. Iustal, Madrid, 2006.

OTERO PASTOR, ISABEL (1998): Evaluación de la calidad ambiental, en: Observatorio Medioambiental, $\mathrm{n}^{\circ} 1$, pp. 15-27, Universidad Complutense de Madrid: Instituto Universitario de Ciencias Ambientales, Madrid.

STANNERS, D.; Y BORDEAU, P. (1995); Europe's environment: the Drobris assessment. European Environment Agency, Brussels, 1995.

TORTOSA, PACO; MIRALLES, BORO; Y LOSTADO, RAFAEL (dirs.) (1996); Glossari de termes ambientals: residus, gestió dels residus, reciclat i residus sòlids urbans. Fundació Bancaixa, Valencia, 1995.

VELÁZQUEZ PATIÑO, ANA CAROLINA (2008): La gestión de los residuos sólidos urbanos en la ciudad de Hannover: un modelo exitoso, en: Anales de Geografía de la Universidad Complutense, vol. 28, $\mathrm{n}^{\circ}$ 1, pp. 163-177, Universidad Complutense de Madrid, Madrid. 